\title{
Original
}

\section{Características del control prenatal y el parto de las mujeres inmigrantes atendidas en el Hospital "Dr. Rafael Ángel Calderón Guardia"}

\author{
(Prenatal care and delivery characteristics of immigrant \\ women at "Dr. Rafael Angel Calderón Guardia" Hospital)
}

Manrique Leal-Mateos, Jorge Barboza-Retana

\section{Resumen}

Servicio de Obstetricia, Hospital Dr. Rafael A. Calderón Guardia Abreviaturas: CCSS, Caja Costarricense de Seguro Social; HCG, Hospital Rafael Ángel Calderón Guardia Correspondencia: Manrique Leal manrique.leal@gmail.com
Justificación y objetivo: El presente estudio tiene como objetivo principal dar a conocer las características más sobresalientes del control prenatal y del parto de la población inmigrante que acude al Servicio de Obstetricia del Hospital Dr. Rafael A. Calderón Guardia, Costa Rica y comparar los resultados obtenidos con los observados en la población costarricense, con el fin de determinar si difieren entre sí.

Materiales y métodos: Se realizó un estudio observacional analítico de corte transversal. Mediante un muestreo consecutivo se analizaron 360 historias clínicas de pacientes gestantes atendidas en HCG entre el 1 de agosto y 7 de septiembre de 2007. Se excluyó del análisis toda paciente que no perteneciera al área de atracción del HCG o que presentara de forma incompleta alguna de las variables de interés. La recolección de la información se realizó mediante una hoja precodificada diseñada con base la que se utiliza para el control prenatal en el sistema de salud nacional.

Resultados: Aproximadamente una de cada 5 pacientes embarazadas que dan a luz en el HCG son inmigrantes. La mayor parte de ellas son de origen nicaragüense, relativamente jóvenes, multíparas y con controles prenatales adecuados. Además, presentan una morbilidad previa e inducida por el embarazo semejante a la encontrada en las gestantes nacionales. Si bien aproximadamente la mitad de las pacientes inmigrantes se captan de forma tardía y presentan una cantidad menor de consultas prenatales a la observada en las embarazadas costarricenses, este aspecto no parece influir en los resultados derivados de la atención de su parto ni en las características de los recién nacidos al momento de su nacimiento

Conclusión: Las características de la población inmigrante que da a luz en el Hospital no son diferentes a las encontradas en las mujeres costarricenses.

Descriptores: Control prenatal, parto, embarazo, inmigrante, Hospital Dr. Rafael A. Calderón Guardia.

\section{Abstract}

Aim: To examine the characteristics of prenatal control and delivery in the immigrant population that gives birth at the "Rafael Angel Calderon Guardia Hospital (HCG), Costa Rica. This study 
ISSN 0001-6002/2008/50/2/107-111 Acta Médica Costarricense, (C2008 Colegio de Médicos y Cirujanos will compare the results obtained within the immigrant population with those of the native Costa Rican population and will determine the differences, if any, between the standard of prenatal care and delivery in the immigrant population and that of the native Costa Rican population.

Materials and methods: This study is a cross-sectional observational study. Three hundred and sixty medical records were analyzed, patients that were treated at the HCG, but did not belong to the hospital's attraction area or had an incomplete record were excluded. The information for the study was collected using a pre-coded information sheet similar to the one used by the country's national health system.

Results: This study demonstrates that immigrant women account for 1 out of every 5 patients giving birth at the HCG. The majority of these immigrants are young, multiparous, Nicaraguan women with adequate prenatal control. The study also shows that the morbidity in the immigrant population is similar to that of the Costa Rican population. It is also noteworthy to mention that half of the immigrant patients began their prenatal control after 13 weeks of gestation, and, had a less prenatal consults than the Costa Rican population. This finding does not seem to have any influence over delivery or neonatal outcomes in the immigrant population.

Conclusion: There are no significant medical differences between the immigrant population that gives birth at the HCG and the native Costa Rican population.

Key words: Prenatal care, delivery, pregnancy, immigrant, Rafael Calderón Guardia Hospital.

Recibido: 1 de octubre de 2007 Aceptado: 15 de enero de 2008
Una de las características poblacionales de la que menos información se tiene en el mundo es la inmigración internacional, por ello, las estimaciones disponibles de los movimientos internacionales de personas pueden diferir considerablemente de la realidad. ${ }^{1}$

Según los informes del Estado de la Nación y diferentes medios de prensa escrita, Costa Rica ha sido el país latinoamericano que cuenta con mayor cantidad de inmigrantes. La mayoría de los extranjeros que residen en la nación son de países de Centro y Suramérica. ${ }^{2-5}$

Lo anterior ha creado una nueva situación sociodemográfica escasamente reconocida por las autoridades del país. Entre todos los aspectos, la asistencia sanitaria de esta población ha sido uno de los retos que más ha cobrado interés en los últimos años. ${ }^{6,7}$

Respecto a este tema, resulta de especial interés la atención que se presta a la mujer embarazada durante su gestación y parto. $\mathrm{Y}$ es que, hasta hace pocos años, la migración era considerada un factor de riesgo para la mujer embarazada, y a las pacientes inmigrantes se les atribuían resultados neonatales adversos y una mayor mortalidad. ${ }^{8,9}$

No obstante, cada vez aparecen más datos en el nivel mundial que evidencian que las gestantes inmigrantes no tienen resultados perinatales tan negativos como cabría esperar por su situación social. Por el contrario, con más frecuenciaseobservanfactoresprotectoresycomportamientos prenatales más saludables que muestran resultados similares, $\mathrm{y}$ en ocasiones, mejores a los observados en gestantes oriundas del país receptor. ${ }^{10-15}$
Dada la escasa evidencia que existe en el Sistema de Salud acerca de esta problemática tema en los últimos años, el presente estudio tiene como objetivo principal dar a conocer las características más sobresalientes del control prenatal y del parto de la población inmigrante que acude al Servicio de Obstetricia del Hospital Dr. Rafael A. Calderón Guardia. Asimismo, pretende comparar los resultados obtenidos con los observados en la población costarricense, con el fin de determinar si difieren entre sí.

\section{Materiales y métodos}

Se realizó un estudio observacional analítico de corte transversal para cumplir con los objetivos del estudio. La presente investigación contó con la aprobación del Comité de Ética del Hospital. Mediante un muestreo consecutivo se analizaron 360 historias clínicas de pacientes gestantes atendidas en el servicio de Obstetricia del Hospital Dr. Rafael A. Calderón Guardia entre el 1 de agosto y el 7 de septiembre de 2007. El tamaño de la muestra se calculó con base en los 5.755 partos atendidos en el Hospital Calderón Guardia durante 2006 y con base en una prevalencia máxima de población inmigrante del $50 \%$. Se estimó un error $\alpha=0,05$ y un error $\beta=0,80$, para lo cuál se utilizó el Sistema Statcalc del programa EpiInfo 2002. Se excluyó del análisis toda paciente que no perteneciera al área de atracción del HCG o que presentara de forma incompleta alguna de las variables de interés. 
La recolección de los datos se efectuó mediante una hoja precodificada diseñada con base en la que se utiliza para el control prenatal en el Sistema de Salud Nacional.

Las variables maternas relacionadas con el control prenatal que se incluyeron fueron: edad en años cumplidos, estado civil, cantidad de embarazos, abortos, cesáreas, morbilidad previa al embarazo, morbilidad inducida por el embarazo, cantidad de consultas de control prenatal, captación tardía de la embarazada por los servicios de salud, ganancia de peso durante la gestación en kilogramos, número de hospitalizaciones durante el embarazo y hemoglobina pre parto.

Las variables relacionadas con el parto que se analizaron fueron: edad gestacional en semanas cumplidas, tipo de parto, realización de episiotomía, presencia de desgarros, presencia de líquido amniótico meconizado.

Las variables neonatales que se estudiaron fueron: sexo, peso al nacer en kilogramos, talla en centímetros, circunferencia cefálica en centímetros, puntuación Apgar al minuto, clasificación según peso/edad gestacional, necesidad de reanimación neonatal, necesidad de hospitalización del recién nacido.

Se utilizó el programa EpiInfo 2002 para el procesamiento de los datos. Las variables cualitativas se analizaron mediante frecuencias y proporciones. Los resultados derivados de las variables cuantitativas se expresaron mediante medidas de tendencia central y de dispersión. La relación entre variables cualitativas se realizó mediante la prueba estadística de Chi cuadrada $\left(\mathrm{X}^{2}\right)$. La diferencia entre promedios y proporciones se comparó mediante la prueba de t Student. Se utilizaron las pruebas exactas de Fisher y la prueba de "U" de Mann-Whitney, cuando fue requerido. El nivel de significancia se fijó en $\mathrm{p} \leq 0.05$. Se utilizó el programa Excel 2007 para la elaboración de tablas.

\begin{tabular}{|lcc|}
\hline \multicolumn{2}{|c|}{$\begin{array}{c}\text { Cuadro 1. Distribución de pacientes } \\
\text { inmigrantes, según país de origen. Estudio } \\
\text { sobre las características del control prenatal y } \\
\text { parto de mujeres inmigrantes, HCG, 2007 }\end{array}$} \\
\hline Nacionalidad & Cantidad & Porcentaje \\
\hline Nicaragua & 58 & 88,0 \\
Colombia & 3 & 4,5 \\
El Salvador & 1 & 1,5 \\
Honduras & 1 & 1,5 \\
Perú & 1 & 1,5 \\
Ecuador & 1 & 1,5 \\
España & 1 & 1,5 \\
\hline Total & 66 & 100 \\
\hline
\end{tabular}

\section{Resultados}

Se analizaron un total de 382 historias clínicas. Se excluyeron del análisis 6 inmigrantes y once nacionales, por no pertenecer al área de atracción del HCG y cuatro extranjeras y una costarricense por no presentar de forma completa alguna variable de interés. Todas las pacientes excluidas fueron sustituidas hasta lograr el tamaño de muestra propuesto inicialmente.

De las 360 pacientes estudiadas, 66 de ellas (18,3\%; IC $95 \%$ 14,6-22,8) fueron identificadas como inmigrantes. El país que mayor cantidad de gestantes extranjeras registró fue Nicaragua con 58 (87,9\%; IC 95\% 80,0-95,8). En el Cuadro 1 se muestran los países de procedencia de cada una de estas pacientes.

Hubo una diferencia significativa entre el promedio de edad de las mujeres extranjeras (27,3 años; $\mathrm{DS} \pm 6,3)$ y el de las nacionales $(25,4$ años $\mathrm{DS} \pm 6,1) ; \mathrm{p}=0,024$. La cantidad de inmigrantes casadas fue de $16(24,2 \%$, IC 95\% 14,5-36,4) y la de costarricenses de 115 (39,1\%, IC 95\% 33,5-44,7); $\mathrm{p}=0,023$. La cantidad primigestas en el grupo de extranjeras fue de $19(28,8 \%$, IC 95\% 18,3-41,3) y en el de costarricenses, de $128(43,5 \%$, IC 95\% 37,8-49,4); $\mathrm{p}=0,027$. Las principales características de la historia obstétrica de las pacientes se muestran en el Cuadro 2.

La cantidad de embarazadas con control prenatal en el grupo de inmigrantes fue de $63(95,5 \%$, IC 95\% 87,3-99,1) y en el de costarricenses, de 287 (97,6\%, IC 95\% 95,2-99,0); $\mathrm{p}=0,333$. Del total de pacientes con control prenatal, la cantidad de gestantes captadas tardíamente en el grupo de extranjeras fue de $36(57,1 \%$, IC 95\% 44,0-69,5) y en el de nacionales, de 76 (26,5\%, IC 95\% 21,5-32,0); p<0,001. En promedio, las inmigrantes fueron captadas a las 16,3 (DS \pm $9,3)$ semanas de gestación y las costarricenses, a las $11, \overline{3}$

\begin{tabular}{|l}
$\begin{array}{c}\text { Cuadro 2. Principales características de la } \\
\text { historia clínica obstétrica de las embarazadas }\end{array}$ \\
inmigrantes y costarricenses. Estudio sobre las \\
características del control prenatal y parto de \\
mujeres inmigrantes, HCG, 2007
\end{tabular}


(DS $\pm 6,5)$ semanas; $p<0,001$. El promedio de consultas prenatales en el grupo de pacientes extranjeras fue de 6,5 (DS $\pm 2,7)$ y en el de nacionales, de 7,8 (DS $\pm 2,5) ; \mathrm{p}<0,001$. Las características más sobresalientes de las pacientes que presentaban control prenatal al momento del parto se muestran en el Cuadro 3.

La edad gestacional en semanas al momento del parto en el grupo de pacientes inmigrantes fue de 38,5 ( $\mathrm{DS} \pm 2,1)$ $y$ en el de costarricenses de 38,4 (DS $\pm 2,2) ; p=0,718$. El porcentaje de partos pretérmino en el grupo de extranjeras fue del $12,1 \%(\mathrm{n}=8$, IC $95 \% 5,4-22,5)$ y del $13,6 \%(\mathrm{n}=40$, IC $95 \% 9,9-18,1)$ en el grupo de nacionales; $p=0,748$.Las características más sobresalientes de la atención del parto se muestran en el Cuadro 4.

El porcentaje de productos masculinos en el grupo de extranjeras fue del $56,1 \%(n=37$, IC $95 \% 43,3-68,3)$ y en el de nacionales del 56,1\% ( $\mathrm{n}=156$, IC 95\% 50,2-61,9); $\mathrm{p}=0,992$. No hubo diferencias estadísticamente significativas entre el peso de los recién nacidos de madres extranjeras $(3076,5$ g. $\pm 619,2)$ y las nacionales $(3153,1$ g. $\pm 562,3)$; $\mathrm{p}=0,326$. No obstante, el porcentaje de neonatos pequeños para edad gestacional fue mayor en el grupo de inmigrantes $(\mathrm{n}=5 ; 7,6 \%$, IC $95 \% 2,5-16,8)$ que en el de costarricenses $(\mathrm{n}=7 ; 2,4 \%$, IC 95\%, 1,0-4,8;); $\mathrm{p}=0,033$. Las características más importantes de los recién nacidos se presentan en el Cuadro 5.

\section{Discusión}

Antes de comentar los resultados obtenidos es importante mencionar algunos factores que pueden influir en su

\begin{tabular}{|c|c|c|c|}
\hline \multicolumn{4}{|c|}{$\begin{array}{c}\text { Cuadro 3. Principales características del control } \\
\text { prenatal de las embarazadas inmigrantes y } \\
\text { costarricenses. Estudio sobre las características } \\
\text { del control prenatal y parto de mujeres } \\
\text { inmigrantes, HCG, } 2007\end{array}$} \\
\hline Característica & $\begin{array}{l}\text { Embarazada } \\
\text { inmigrante }\end{array}$ & $\begin{array}{l}\text { Embarazada } \\
\text { costarricense }\end{array}$ & $p$ \\
\hline Control prenatal (\%) & 95,5 & 97,6 & 0,333 \\
\hline Captación tardía (\%) & 57,1 & 26,5 & $<0,001$ \\
\hline $\begin{array}{l}\text { Semana de captación } \\
\text { (promedio) }\end{array}$ & 16,3 & 11,3 & $<0,001$ \\
\hline $\begin{array}{l}\text { Número de consultas } \\
\text { prenatales (promedio) }\end{array}$ & 6,5 & 7,9 & $<0,001$ \\
\hline $\begin{array}{l}\text { Mas de } 5 \text { consultas } \\
\text { prenatales }\end{array}$ & 72,7 & 87,4 & 0,002 \\
\hline $\begin{array}{l}\text { Ganancia de peso ( } \mathrm{Kg} \\
\text { promedio) }\end{array}$ & 10,1 & 11,3 & 0,058 \\
\hline $\begin{array}{l}\text { Hospitalización previa } \\
\text { al parto }(\%)\end{array}$ & 7,6 & 11,9 & 0,311 \\
\hline $\begin{array}{l}\text { Hemoglobina preparto } \\
\text { (g/dl promedio) }\end{array}$ & 12,7 & 12,8 & 0,963 \\
\hline
\end{tabular}

interpretación. Primeramente, el estudio no mide causalidad, solo relación o asociación entre las variables independientes y dependientes seleccionadas. Por otro lado, al ser un estudio de base hospitalaria y no poblacional, los resultados son aplicables únicamente a las pacientes atendidas en el HCG. Por último, al utilizar historias clínicas de los expedientes médicos de las pacientes como fuente de datos, el estudio no se encuentra libre de sesgos de información.

Con base este concepto, el estudio demostró que aproximadamente una de cada cinco pacientes embarazadas que dan a luz en el HCG son inmigrantes. La mayor parte de ellas son de origen nicaragüense, relativamente jóvenes, multíparas y con controles prenatales adecuados. Además, presentan una morbilidad previa e inducida por el embarazo semejante a la encontrada en las gestantes nacionales. Si bien aproximadamente la mitad de las pacientes inmigrantes se captan de forma tardía y presentan una cantidad menor de consultas prenatales a la observada en las embarazadas costarricenses, este aspecto no parece influir en los resultados derivados de la atención de su parto, ni en las características de los recién nacidos al momento de su nacimiento. El peso, la talla y la circunferencia cefálica de los hijos de madres extranjeras no parece diferir de los encontrados en aquellos de madres costarricenses. Es importante resaltar que el porcentaje de neonatos pequeños para edad gestacional fue mayor en el grupo de madres inmigrantes, pero la necesidad de reanimación neonatal y hospitalización fue semejante en ambos grupos. Por tanto, se puede concluir que las características de la población inmigrante que da a luz en el Hospital no son diferentes a las encontradas en las mujeres costarricenses. Si bien existen diferencias en lo concerniente a su control prenatal, estas resultan más del ámbito social que del médico y es quizás aquí a donde deberían redirigirse los esfuerzos de las autoridades de salud para mejorar la situación de estas pacientes.

\begin{tabular}{|c|c|c|c|}
\hline $\begin{array}{r}\text { Cuadro 4. Prin } \\
\text { la atención del } \\
\text { inmigrantes y cost } \\
\text { características de } \\
\text { mujeres in }\end{array}$ & $\begin{array}{l}\text { cipales car } \\
\text { parto de la } \\
\text { arricenses } \\
\text { l control p } \\
\text { migrantes, }\end{array}$ & $\begin{array}{l}\text { cterísticas } \\
\text { embarazad } \\
\text { Estudio sob } \\
\text { enatal y par } \\
\text { HCG, } 2007\end{array}$ & $\begin{array}{l}\text { le } \\
\text { as } \\
\text { re las } \\
\text { o de }\end{array}$ \\
\hline Característica & $\begin{array}{c}\text { Embarazada } \\
\text { inmigrante }\end{array}$ & $\begin{array}{l}\text { Embarazada } \\
\text { costarricense }\end{array}$ & $p$ \\
\hline $\begin{array}{l}\text { Edad gestacional en } \\
\text { semanas (promedio) }\end{array}$ & 38,5 & 38,4 & 0,718 \\
\hline Pretérmino (\%) & 12,1 & 13,6 & 0,748 \\
\hline Parto vaginal (\%) & 78,8 & 85,7 & 0,161 \\
\hline Cesárea (\%) & 21,2 & 14,3 & 0,161 \\
\hline Liquido meconizado (\%) & 20,7 & 25,8 & 0,372 \\
\hline 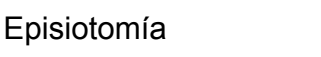 & 38,5 & 28,8 & 0,189 \\
\hline Desgarro perineal & 30,8 & 32,1 & 0,846 \\
\hline
\end{tabular}




\begin{tabular}{|c|c|c|c|}
\hline $\begin{array}{r}\text { Cuadro 5. Pri } \\
\text { los recién na } \\
\text { inmigrantes y cos } \\
\text { características } d \\
\text { mujeres in }\end{array}$ & $\begin{array}{l}\text { cipales cara } \\
\text { idos de las } \\
\text { arricenses. } \\
\text { l control pr } \\
\text { migrantes, }\end{array}$ & $\begin{array}{l}\text { Icterísticas c } \\
\text { embarazada } \\
\text { Estudio sob } \\
\text { enatal y part } \\
\text { HCG, } 2007\end{array}$ & $\begin{array}{l}\text { e } \\
\text { re las } \\
\text { o de }\end{array}$ \\
\hline Característica & $\begin{array}{l}\text { Embarazada } \\
\text { inmigrante }\end{array}$ & $\begin{array}{l}\text { Embarazada } \\
\text { costarricense }\end{array}$ & $p$ \\
\hline Sexo masculino (\%) & 56,1 & 56,1 & 0,992 \\
\hline $\begin{array}{l}\text { Peso al nacer en } \\
\text { gramos (promedio) }\end{array}$ & 3076,5 & 3153,1 & 0,326 \\
\hline $\begin{array}{l}\text { Talla en centímetros } \\
\text { (promedio) }\end{array}$ & 48,9 & 49,1 & 0,626 \\
\hline $\begin{array}{l}\text { Circunferencia } \\
\text { cefálica en } \\
\text { centímetros } \\
\text { (promedio) }\end{array}$ & 33,5 & 34,7 & 0,567 \\
\hline $\begin{array}{l}\text { Puntuación Apgar }<7 \\
\text { al minuto (\%) }\end{array}$ & 12,1 & 8,5 & 0,357 \\
\hline $\begin{array}{l}\text { Clasificación Peso/ } \\
\text { Edad }\end{array}$ & & & \\
\hline $\begin{array}{l}\text { Pequeño para edad } \\
\text { gestacional }(\%)\end{array}$ & 7,5 & 2,1 & 0,033 \\
\hline $\begin{array}{l}\text { Adecuado para edad } \\
\text { gestacional }(\%)\end{array}$ & 80,3 & 86,2 & 0,236 \\
\hline $\begin{array}{l}\text { Grande para edad } \\
\text { gestacional }(\%)\end{array}$ & 12,2 & 11,7 & 0,898 \\
\hline $\begin{array}{l}\text { Necesidad de reani- } \\
\text { mación neonatal (\%) }\end{array}$ & 21,2 & 24,1 & 0,611 \\
\hline $\begin{array}{l}\text { Necesidad de } \\
\text { hospitalización } \\
\text { neonatal }(\%)\end{array}$ & 13,6 & 14,6 & 0,836 \\
\hline
\end{tabular}

En Costa Rica los servicios de salud se encuentran disponibles para quien los necesite, para los inmigrantes el acceso a estos servicios depende principalmente de su condición migratoria y sus condiciones de contratación laboral. ${ }^{16}$

\section{Referencias}

1. Organización Panamericana de la Salud. Volumen 1: La Salud en las Américas, Edición de 2002. Washington D.C.: OPS, 2002.

2. Proyecto Estado de la Nación en Desarrollo Humano Sostenible. Estado de la Nación en desarrollo sostenible: Cuarto informe. San José, Costa Rica: Proyecto Estado de la Nación, 1997.

3. Proyecto Estado de la Nación en Desarrollo Humano Sostenible. Estado de la Nación en desarrollo sostenible: Quinto informe. San José, Costa Rica: Proyecto Estado de la Nación, 1998.

4. Proyecto Estado de la Nación en Desarrollo Humano Sostenible. Estado de la Nación en desarrollo sostenible: Octavo informe. San José, Costa Rica: Proyecto Estado de la Nación, 2001.

5. Soza, $\mathrm{C}$ y Naudon, $\mathrm{M}$. Inmigrantes latinos buscan nuevos horizontes. La Nación 2003, miércoles 30 de abril; sección "Economía": 27-A .

6. Rosero L. ¿20\% de inmigrantes? La Nación 2007, sábado 19 de mayo; sección "Opinión": 37-A.

7. Herdocia M. Ticos y nicas: Somos hermanos. La Nación 2007, domingo 01 de julio; sección “Opinión”: 35-A.

8. Essen B, Bodker B, Sjoberg NO, Langhoff-Ross J, Greisen G, Gudmundsson $\mathrm{S}$ et al. Are some perinatal deaths in immigrant groups linked to suboptimal perinatal care service? BJOG. 2002; 109: 677682.

9. Diani F, Zanconato G, Foschi F, Turinetto A, Franchi M. Management of the pregnant immigrant women in the decade 1992-2001. Int $J$ Obstet Gynaecol. 2003; $83:$ 257-265.

10. Gaffney K. Perinatal risk factor among foreing-born central american women: A comparative study. Public Health Nurs. 2000; 17: 415422.

11. Martín I, López M, Lozano J, Mur A. Resultados perinatales de las gestantes inmigrantes. An Pediátr. 2006; 64: 550-556.

12. Sánchez Y, Muñoz N, Pérez S, Robledo A, Pallás CR, De la Cruz J et al. Mujeres inmigrantes: Características del control prenatal y del parto. Prog Obstet Ginecol. 2003; 46: 441-447.

13. Pérez S, Muñoz N, Robledo A, Sánchez Y, Pallás CR, De la Cruz J. Características de las mujeres inmigrantes y de sus hijos recién nacidos. An Pediátr. 2004; 60: 3-8.

14. Mc Glade M, Saha S, Dahistrom MA. The latina paradox: An opportunity for restructuring prenatal care delivery. Am J Public Health. 2004; 94: 2062-2065.

15. Im EO, Yang K. Theories on Immigrant Women's Health. Health Care Women Int. 2006; 27:666-681.

16. Organización Panamericana de la Salud. Migración y salud en Costa Rica: Elementos para su análisis. San José Costa Rica: OPS; 2003 